\title{
Ab initio studies of isolated hydrogen vacancies in graphane
}

\author{
R. E. Mapasha* \\ Department of Physics, University of Pretoria, Pretoria 0002, South Africa \\ M. P. Molepo \\ College of Graduate Studies, University of South Africa, UNISA 0003 Pretoria, South \\ Africa \\ N. Chetty \\ Department of Physics, University of Pretoria, Pretoria 0002, South Africa \\ National Institute for Theoretical Physics, Johannesburg, 2000, South Africa
}

\begin{abstract}
We present a density functional study of various hydrogen vacancies located on a single hexagonal ring of graphane (fully hydrogenated graphene) considering the effects of charge states and the position of the Fermi level. We find that uncharged vacancies that lead to a carbon sublattice balance are energetically favourable and are wide band gap systems just like pristine graphane. Vacancies that do create a sublattice imbalance introduce spin polarized states into the band gap, and exhibit a half-metallic behavior with a magnetic moment of $1.00 \mu_{B}$ per vacancy. The results show the possibility of using vacancies in graphane for novel spin-based applications. When charging such vacancy configurations, the deep donor $(+1 / 0)$ and deep ac-
\end{abstract}

\footnotetext{
*Corresponding author

Email address: edwin.mapasha@up.ac.za (R. E. Mapasha)
} 
ceptor $(0 /-1)$ transition levels within the band gap are noted. We also note a half-metallic to metallic transition and a significant reduction of the induced magnetic moment due to both negative and positive charge doping.

Keywords: vacancies, sublattice, density functional theory, charge state, magnetic moment

\section{Introduction}

Free standing graphene, a single layer of graphite, was first synthesized in 2004. [1] It has very special properties such as half integer quantum Hall effect and high charge carrier mobility due to linear dispersion at the Dirac point. [2, 3, 4, 5] Also, ballistic transport capability over long displacement at room temperatures makes graphene a suitable candidate for electronic applications. [2, 3, 4, 5] However, a lack of band gap in the electronic spectrum of graphene [3] hinders its direct integration in the electronic devices. To solve this problem, much research has focused on altering its physical and chemical properties to engineer its band gap.

Several techniques such as cutting graphene into nanoribbons $[6,7,8]$, creating Stone-Wales-type defects $[9,10,11,12,13,14,15]$ and chemical functionalization $[16,17,18,19,20,21,22,23,24,25,26,27,28,29]$ using impurities such as hydrogen and fluorine atoms have been considered. Both theoretical and experimental studies demonstrated that full hydrogenation $[28,29]$ and fluorination $[30,31]$ of graphene result in new thermodynamically stable crystalline materials known as graphane and fluorographene respectively, and the process is also reversible. [29, 31, 32] It must be mentioned that graphane was first proposed by Sluiter et al. [33] using cluster 
expansion method, before the isolation of graphene. [1] Graphane is a wide band gap semiconductor, with a band gap of $3.50 \mathrm{eV}$ according to theoretical results. [28]

The chair configuration of graphane is the most energetically stable isomer as compared to boat and armchair. [28, 33, 34] An overview of other graphane isomers can be found in the review article by Sahin et al. [35] and references therein. The creation of various defects in graphane significantly alters its electronic and magnetic behaviour. [35] First-principles investigations have revealed that the presence of $\mathrm{H}$ vacancy defects in graphane leads to a semiconductor-metallic transition. [36] Using the GW method, Lebegue et al. [19] reported that a single $\mathrm{H}$ vacancy defect in graphane provide some impurity states in the band gap at $2 \mathrm{eV}$ above the valence-band maximum. They also confirmed this behaviour from the measurements of optical conductivity of non-stoichiometric graphane. Density functional theory (DFT) studies reported that the creation of this $\mathrm{H}$ vacancy defect induces a total magnetic moment of $1.0 \mu B$. [37, 38, 39] In the case of configurations with more than one $\mathrm{H}$ vacancy defect, it has been shown that for odd numbers of nearest-neighbour vacancies, there is always one unpaired electron inducing a magnetic moment of $1.0 \mu B$. [36, 37, 39, 40] For even numbers of nearestneighbour vacancies, there is complete pairing of electrons producing no magnetic moment. [36, 39, 40, 41] Wu et al [39] have also shown that $\mathrm{H}$ vacancies in the line configurations that are not adjacent to each other gives number of magnetic moments equal to the number of vacancies. These studies have shown that the presence of $\mathrm{H}$ vacancy defects in graphane could be useful for future data storage and spintronics applications. Despite the availability of 
rich literature focusing on $\mathrm{H}$ vacancy defects in graphane, studies that takes into account the effects of charge doping are still lacking. Charge doping, the addition of electrons or holes, usually affects the electronic structure and magnetic properties, in particular the conductivity of a material. Therefore, it is worth investigating the altering of the vacancy-induced ferro states within the band gap due to charge doping. The prediction of the thermodynamic stability through the formation energies and thermodynamic transition levels within the band gap of electron or hole doped graphane with $\mathrm{H}$ vacancy defects, and the examination of their associated magnetic properties can give another domain for spin based electronic applications.

In this work, we systematically investigate the energetics, electronic and magnetic properties of graphane with various hydrogen vacancy configurations located on a single hexagonal ring under the influence of charge doping, using DFT approach. The effect of charge doping on the position of $\mathrm{H}$ vacancy defect induced donor states is investigated. We note that -1 charged (+1 charged) vacancy configurations are more energetically favourable in the p-type (n-type) region respectively. The addition of charge state $q=-1(+1)$ shifts the induced donor states as well as the Fermi level toward the CBM (VBM). It was also noted that an induced magnetic moment is always reduced by charge doping.

\section{Computational details}

The ground state electronic structure calculations were performed using density functional theory formalism implemented within the Vienna $a b$ initio simulation package (VASP) code. [42] For the exchange-correlation 
interaction, we used the generalized gradient approximations (GGA) parameterization of Perdew, Burke and Ernzerhof (PBE). [43] The spin polarization was included for all calculations. For the core-electron interactions, the pseudopotentials (with $2 \mathrm{~s}^{2} 2 \mathrm{p}^{2}$ and $1 \mathrm{~s}^{1}$ valence electrons of $\mathrm{C}$ and $\mathrm{H}$ atoms, respectively) were generated using projector augmented wave (PAW) methods. [44] An energy cut-off of $500 \mathrm{eV}$ was set for the plane wave functions expansion. We performed a convergence test of our results for total energy differences on the $(1 \times 1)$ unit cell, and concluded that a grid of size $10 \times 10 \times 1$ generated using Monkhorst-Pack scheme [45] is sufficiently converged, for accurate sampling of the Brillouin zone. The total energies were converged to within $10^{-7} \mathrm{eV}$.

The Methfessel-Paxton (MP) scheme [46] with a smearing width of 0.2 $\mathrm{eV}$ was used to populate electronic states in the self-consistent field calculations. The Hellman-Feynman theorem was used to calculate the atomic forces, whereby the atomic positions were allowed to relax until the forces were reduced to less than $0.01 \mathrm{eV} \AA^{-1}$. The separation for the graphane layers in the supercell was set to $15 \AA$ to avoid the interlayer spurious interactions in the periodic system.

In this study, we considered the investigation of graphane system with various isolated hydrogen vacancies on a single hexagonal ring of a $7 \times 7$ supercell. The total energy was converged with respect to the supercell size containing the largest vacancy defect considered. The different supercell sizes considered are $3 \times 3,5 \times 5,7 \times 7$ and $9 \times 9$ corresponding to 32, 50, 98 and 168 carbon atoms. The calculated formation energy for the largest $\mathrm{H}$ vacancy defect was calculated for each supercell size. Our calculated formation 
energy is $2.093 \mathrm{eV}, 2.419 \mathrm{eV}, 2.547 \mathrm{eV}$ and $2.556 \mathrm{eV}$ corresponding to $3 \times 3$, $5 \times 5,7 \times 7$ and $9 \times 9$ supercell size respectively. It is noted that the formation energy difference between $7 \times 7$ and $9 \times 9$ supercell sizes converges to $9 \mathrm{meV}$, thus a $7 \times 7$ supercell was further used for the various vacancy defects calculations.

\section{Results and discussion}

\subsection{Hydrogen vacancies}

The study was conducted on a thermodynamically stable form of graphane (chair-like-graphane) [28,33], where the pattern of hydrogenation is always $\mathrm{H}$ above $(a)$ and below $(b)$ the graphene plane in an alternating manner. The number of hydrogen vacancies in a single ring of graphane ranges from one to six. The carbon atoms labeled 1 through 6 on Fig 1, present the sites in which the hydrogen atoms can be removed, and thus this also enables us to name and distinguish the identified configurations. For instance, configuration $c_{1}$ is named $V 1_{a}$ which denotes a hydrogen vacancy above carbon site 1. For two hydrogen vacancies, three unique configurations $\mathrm{c}_{2}, \mathrm{c}_{3}$ and $\mathrm{c}_{4}$ are identified and denoted as $\mathrm{V} 1_{a} 2_{b}$ (ortho), $\mathrm{V} 1_{a} 3_{a}$ (meta) and $\mathrm{V} 1_{a} 4_{b}$ (para), respectively. In so doing, a total of 12 unique hydrogen vacancy configurations on a single hexagonal ring of graphane are identified and presented in Table 1. The formation energies and thermodynamic charge transition levels for these configurations are discussed in detail in the next subsection.

\subsection{Hydrogen vacancy formation energies and charge transition levels}

To compare the thermodynamic stability of the uncharged vacancy configurations presented in Table 1, their formation energies $E_{f}\left(V_{H}\right)$ are calculated 


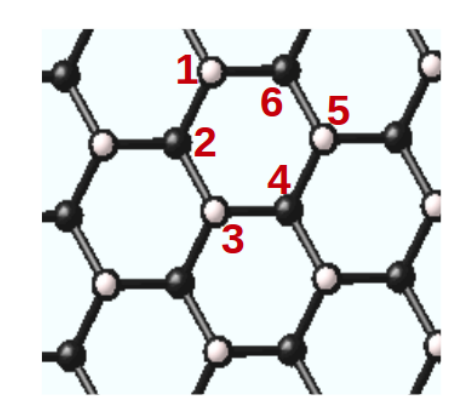

Figure 1: The atomic structure of graphane. The black and white spheres represent carbon and hydrogen atoms respectively. The numbers 1-6 indicate the hydrogen vacancy sites on an isolated hexagonal ring. The hydrogen atoms on the even numbered sites are attached below the layer. 
Table 1: The isolated hydrogen vacancy sites from a $7 \times 7$ supercell of graphane monolayer.

\begin{tabular}{ccc}
\hline \hline Configurations & Vacancy sites & No of vacancies \\
\hline$c_{1}$ & $\mathrm{~V} 1_{a}$ & 1 \\
$c_{2}$ & $\mathrm{~V} 1_{a} 2_{b}$ & 2 \\
$c_{3}$ & $\mathrm{~V} 1_{a} 3_{a}$ & 2 \\
$c_{4}$ & $\mathrm{~V} 1_{a} 4_{b}$ & 2 \\
$c_{5}$ & $\mathrm{~V} 1_{a} 2_{b} 3_{a}$ & 3 \\
$c_{6}$ & $\mathrm{~V} 1_{a} 2_{b} 4_{b}$ & 3 \\
$c_{7}$ & $\mathrm{~V} 1_{a} 3_{a} 5_{a}$ & 3 \\
$c_{8}$ & $\mathrm{~V} 1_{a} 2_{b} 3_{a} 4_{b}$ & 4 \\
$c_{9}$ & $\mathrm{~V} 1_{a} 2_{b} 3_{a} 5_{a}$ & 4 \\
$c_{10}$ & $\mathrm{~V} 1_{a} 2_{b} 4_{b} 5_{a}$ & 4 \\
$c_{11}$ & $\mathrm{~V} 1_{a} 2_{b} 3_{a} 4_{b} 5_{a}$ & 5 \\
$c_{12}$ & $\mathrm{~V} 1_{a} 2_{b} 3_{a} 4_{b} 5_{a} 6_{b}$ & 6 \\
\hline \hline
\end{tabular}


using the following equation:

$$
E_{f}\left(V_{H}\right)=E_{t o t}\left(V_{H}\right)-E_{t o t}(G)+n_{H} \mu_{H} .
$$

The terms $E_{t o t}\left(V_{H}\right)$ and $E_{t o t}(G)$ are the total energies of graphane with hydrogen vacancies, and without vacancies respectively. The term $\mu_{H}$ refers to the hydrogen chemical potential calculated as the converged energy of isolated $\mathrm{H}_{2}$ molecule in a large cell and $n_{H}$ is the number of hydrogen atoms in a supercell. The calculated formation energies as a function of various configurations of hydrogen vacancies are presented in Fig. 2a.
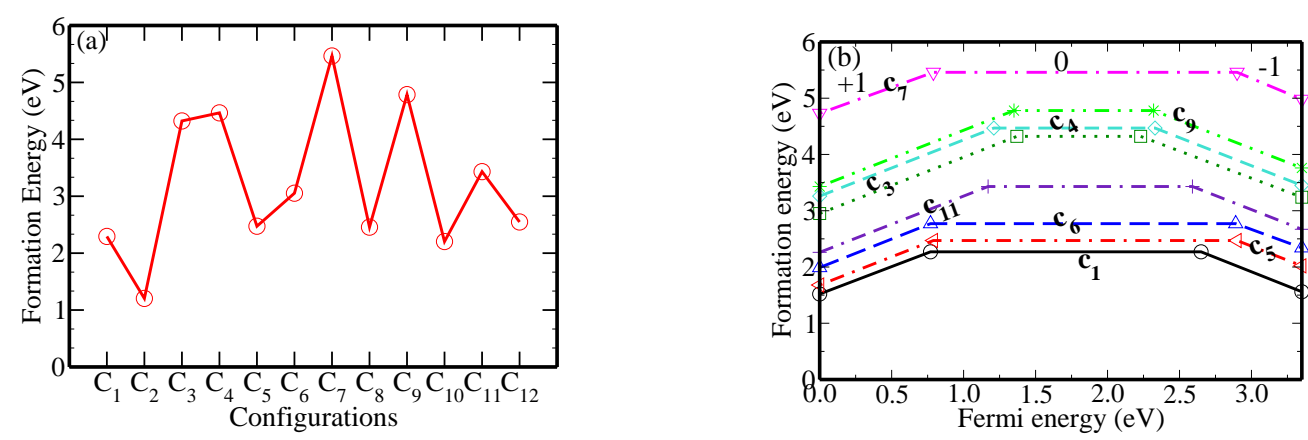

Figure 2: (a) The calculated formation energies of uncharged unique configurations of hydrogen vacancies in graphane. (b) Formation energies of unique configurations of hydrogen vacancies as a function of the Fermi level within the graphane band gap, for different charge states $(+1,0,-1)$. We only show the line segments that correspond to the lowest energy charge states. Geometric shapes in the curves indicate the transitions between the two charge states

We see in Fig. 2a that the formation energies of various hydrogen vacancies in graphane are positive (endothermic), which is an indication that they may not easily form. This is in contrast with the case of graphene hydro- 
genation where the formation energies are negative [28] with graphene taken as a reference. In the case of hydrogen di-vacancy, the ortho configuration $c_{2}(1.16 \mathrm{eV})$ is more energetically favourable than the meta $c_{3}(4.32 \mathrm{eV})$ and the para $c_{4}(4.46 \mathrm{eV})$. Dzhurakhalov et al. [47] studied the chemisorption of the ortho, meta and para configurations of hydrogen isomers on graphene monolayer and reported the same stability trend. Our obtained formation energy value for $c_{3}$ agrees very well with that reported by $\mathrm{Wu}$ et al. [39]. In agreement with the previous study [36], it is clear that the formation energy of each vacancy configuration greatly depends on the carbon sublattice in which hydrogen vacancy is created. For instance, in vacancy configuration $\mathrm{c}_{2}$ (ortho vacancy isomer), the two hydrogen atoms removed from sites 1 and 2 leave a single localized electron on respective carbon atoms. Since the two carbon atoms (C1 and $\mathrm{C} 2)$ are bonded together, their two localized electrons pair to form a $\pi$-bond that minimizes the total energy of the system, and this is considered to be sublattice balance. References [18, 48] mentioned that the energetically favourable structures are those that minimize or avoid the sublattice imbalance during hydrogenation. In configurations $c_{3}$ and $c_{4}$ which are meta and para $\mathrm{H}$ vacancy isomers respectively, the pairing of localized electrons is not possible, thus there is a high possibility of sublattice imbalance which result in these di-vacancy configurations having relatively high formation energies.

The issue of sublattice imbalance still plays a role in tri-vacancy configurations $\left(c_{5}, c_{6}\right.$ and $\left.c_{7}\right)$. Comparing these configurations, it is seen in Fig. 2a that $c_{5}$ and $c_{6}$ have low formation energies of $2.47 \mathrm{eV}$ and $3.01 \mathrm{eV}$ respectively, whereas $c_{7}$ is significantly high with the value of $5.46 \mathrm{eV}$. In con- 
figurations $c_{5}$ and $c_{6}$ there is a possibility of one unpaired electron localized around carbons $\mathrm{C}_{3}$ and $\mathrm{C}_{4}$ respectively because those belonging to $\mathrm{C}_{1}$ and $\mathrm{C}_{2}$ do pair, whereas in configuration $\mathrm{c}_{7}$ the localized electrons belonging to three vacancy sites $\mathrm{C}_{1}, \mathrm{C}_{3}$ and $\mathrm{C}_{5}$ cannot pair (see Table 1 ). The relatively high formation energy obtained for $c_{7}$ is indicative of its failure to avoid or reduce the sublattice imbalance.

In the case of tetra-vacancy configurations, we observe in Fig. 2a that configurations $c_{8}$ and $c_{10}$ are about $2.30 \mathrm{eV}$ lower in formation energy than configuration $c_{9}$. In structures $c_{8}$ and $c_{10}$ which have di-ortho vacancy isomers, an even number of hydrogen vacancies are created in a contiguous sequence around a hexagonal ring, hence the sublattice is always balanced. Although $c_{9}$ has even number of vacancies, there is no complete pairing of electrons because it contains both ortho and meta vacancy isomers, and this results in sublattice imbalance. Configuration $c_{12}$ is competing with those other configurations that have the lowest formation energy due to compact of six $\mathrm{H}$ vacancies surrounding the hexagonal ring (tri-ortho vacancy isomer). It is clear that the vacancy induced localized electrons pair for the entire hexagonal ring and resulting in the structure having all the sublattice balanced. This in agreement with Chandrachud et al. [36] who reported that the structure having a compact form of vacancies has the highest binding energy.

It was reported from the literature that the configurations that have sublattice imbalance (meta and para $\mathrm{H}$ vacancy defect isomers) are those that introduce donor states within the graphane band gap. [36, 37, 39, 40, 41] Although energetically unfavourable, they are promising candidates for elec- 
tronic applications due to the observed interesting electronic and ferro magnetic properties. [36, 37, 39, 40, 41] Therefore, these particular systems deserve further investigations. We learned from previous discussions that those configurations are $\mathrm{c}_{1}, \mathrm{c}_{3}, \mathrm{c}_{4}, \mathrm{c}_{5}, \mathrm{c}_{6}, \mathrm{c}_{7}, \mathrm{c}_{9}$ and $\mathrm{c}_{11}$. We therefore manipulate the induced $\mathrm{H}$ vacancy donor states by adding (removing) an electron state to (from) these vacancy configurations. This also affects their energetics, electronic and magnetic properties.

In DFT calculations, charge doping is performed by applying an opposite background charge to keep the system neutral. Addition of an electron in a $7 \times 7$ graphane supercell corresponds to a charge concentration of approximately $3.89 \times 10^{13} / \mathrm{cm}^{2}$, whereas the removal of an electron creates a hole corresponding to $-3.89 \times 10^{13} / \mathrm{cm}^{2}$. The experimental carrier concentration of the order of $10^{12} / \mathrm{cm}^{2}$ was reported by Elias et al. [29] for graphane. Although our calculated carrier concentration values are relatively higher, they can still be accessible as it has been shown that charge carriers in graphene can be tuned to concentrations as high as $10^{13} / \mathrm{cm}^{2}$ under ambient conditions. [2]

We calculate the formation energy for each vacancy configuration in the presence of charge state $q$ using this equation

$$
E_{f}\left(V_{H}^{q}\right)=E_{t o t}\left(V_{H}^{q}\right)-E_{t o t}(G)+n_{H} \mu_{H}+q\left(E_{F}+\epsilon_{v}\right),
$$

where $E_{\text {tot }}\left(V_{H}^{q}\right)$ is the total energy of the supercell having the hydrogen vacancies in a charge state $q, n_{H}$ is the number of hydrogen atoms removed from the supercell and $\mu_{H}$ refers to the hydrogen chemical potential. $E_{F}$ is the Fermi energy also referred to as the electron chemical potential measured 
relative to the valence band maximum (VBM) $\epsilon_{v}$ of graphane.

Markov and Payne [49] mentioned that the electrostatic interaction between periodic array of defects makes the energy of the supercell to converge very slowly as a function of supercell size and also introduces total energy errors. It is also expected that these errors may arise during the charge injection. In this work, Markov-Payne [49] correction term is included during the calculations of the formation energies. To determine thermodynamic transition energy levels between charge states $q$ and $q^{\prime}$, we use the following expression [50];

$$
\varepsilon\left(q / q^{\prime}\right)=\frac{E_{f}\left(V_{H}^{q}\right)-E_{f}\left(V_{H}^{q^{\prime}}\right)}{q^{\prime}-q}
$$

where $E_{f}\left(V_{H}^{q}\right)$ and $E_{f}\left(V_{H}^{q^{\prime}}\right)$ are the formation energies of the hydrogen vacancy configuration in the charge states $q$ and $q^{\prime}$ when the position of the Fermi level is exactly at the VBM, respectively. In principle, the transition energy level $\varepsilon\left(q / q^{\prime}\right)$ is the position of Fermi-level in which the formation energies of vacancies charged $q$ and $q^{\prime}$ are equal. In this study, the +1 and -1 charge states of hydrogen were considered. The calculated formation energies of various hydrogen vacancy configurations in three charge states $(q=+1,0$, -1) as a function of the Fermi level that is constrained within the band gap $(3.34 \mathrm{eV})$ of graphane are presented in Fig. 2b. This band gap was calculated using quasi particle method $[51,52]$ as the energy difference between ionization potential and electron affinity. The ionization energy is calculated with reference to CBM and the electron affinity with reference to VBM.

The removal or addition of an electron lowers the formation energies of these vacancies as follows: as the $E_{F}$ increases, +1 charged vacancies show increase in formation energies, while the formation energies of - 1 charged va- 
cancies decrease monotonously. As expected, it is found that the formation energies of uncharged hydrogen vacancy configurations are constant irrespective of the change in $E_{F}$. Comparing vacancy configurations, Fig. 2b shows that $\mathrm{V} 1_{a}$ is consistently most energetically favourable followed by $\mathrm{V} 1_{a} 2_{b} 3_{a}$ and $\mathrm{V} 1_{a} 2_{b} 4_{b}$. These configurations have one localized electron around the dehydrogenated carbon in the neutral state as mentioned earlier. The slight differences in their formation energies might be due to the effect of the number of vacancies created and the sublattices in which the hydrogen atoms are removed. For instance, configurations $\mathrm{V} 1_{a} 2_{b} 3_{a}$ and $\mathrm{V} 1_{a} 2_{b} 4_{b}$ differ only in hydrogen vacancy sublattices. Adding $(q=-1)$ or removing $(q=+1)$ an electron to or from these configurations, $\mathrm{V} 1_{a} 2_{b} 3_{a}$ is slightly higher in energy than $\mathrm{V} 1_{a} 2_{b} 4_{b}$ as Fermi energy increases. Configuration $\mathrm{V} 1_{a} 3_{a} 5_{a}$ has three number of localized unpaired electrons around the dehydrogenated carbon atoms, although the charge injection slightly reduced its formation energy, it is the most energetically unfavourable configuration.

The notable difference in the behavior of hydrogen vacancy configurations due to charge doping is the variation of thermodynamic transition levels (Donor and acceptor levels) within the band gap (see Table 2). The donor levels are evaluated relative to VBM, while the acceptor levels are evaluated relative to CBM. It is seen in Fig. $2 \mathrm{~b}$ that hydrogen vacancies exhibit both donor $(+1 / 0)$ and acceptor $(0 /-1)$ transition levels within the band gap. It is evident that the properties of hydrogen vacancies in graphane would greatly depend on their charge states. Starting with configurations that have odd number of unpaired electrons localized around the dehydrogenated carbon atoms, we note that configuration $c_{1}$ possesses deep donor transition level 
Table 2: Thermodynamic transition energies (in $\mathrm{eV}$ ) of various configurations of hydrogen vacancies in graphane. Energies are measured relative to the $\operatorname{VBM}\left(E_{v}\right)$ or $\mathrm{CBM}\left(E_{c}\right)$.

\begin{tabular}{ccc}
\hline \hline Configurations & $(+1 / 0)$ & $(0 /-1)$ \\
\hline $\mathrm{c}_{1}$ & $E_{v}+0.77$ & $E_{c}-0.70$ \\
$\mathrm{c}_{3}$ & $E_{v}+1.37$ & $E_{c}-1.12$ \\
$\mathrm{c}_{4}$ & $E_{v}+1.21$ & $E_{c}-1.02$ \\
$\mathrm{c}_{5}$ & $E_{v}+0.79$ & $E_{c}-0.45$ \\
$\mathrm{c}_{6}$ & $E_{v}+0.79$ & $E_{c}-0.32$ \\
$\mathrm{c}_{7}$ & $E_{v}+0.79$ & $E_{c}-0.45$ \\
$\mathrm{c}_{9}$ & $E_{v}+1.35$ & $E_{c}-1.03$ \\
$\mathrm{c}_{11}$ & $E_{v}+1.17$ & $E_{c}-0.79$ \\
\hline \hline
\end{tabular}


$(0 /+1)$ at $E_{v}+0.77 \mathrm{eV}$, followed by $\mathrm{c}_{5}$ and $\mathrm{c}_{6}$ having the same level at position $E_{v}+0.79 \mathrm{eV}$. On the other hand, these configurations also possess the deep acceptor levels related to $(0 /-1)$ transition shown in Table 2 . We see that configuration $\mathrm{c}_{7}$ having three unpaired localized electrons possesses acceptor and donor levels associated with $(0 /-1)$ and $(0 /+1)$ further away from the middle of the gap. Configurations $c_{3}, c_{4}$ and $c_{9}$ that have two unpaired electrons still possess acceptor and donor levels within the band gap (see Fig. 2b). We therefore suggest that the charge states in hydrogen vacancy systems can easily be affected by the change in positions of the Fermi level. Although our DFT transition level values might not agree well with the experimental values owing to its weaknesses in predicting the band gaps [53], we suggest that the experimental characterization of these vacancies might depict two observable peaks associated with $(+1 / 0)$ and $(0 /-1)$ transition level. These deep levels also imply that the peaks will appear at high temperature, and this may render such kind of systems inappropriate for room temperature devices applications. For higher vacancy concentrations, the disorder of the vacancies would introduce different positions in the donor states within the band gap which would be more easily accessible at lower temperatures by electron tunneling between the in-gap states.

\subsection{Electronic and magnetic properties}

Fig. 3 depicts spin polarized total density of states for pristine graphane. It is seen that the majority density of states are invertedly symmetrical to minority density of states for the entire plot, indicating non-spin polarization in this system. Basically, in graphane, the bonding network around each and every carbon atom is no longer in $\mathrm{sp}^{2}$ but $\mathrm{sp}^{3}$ hybridization. [29, 54, 55] 
The measurable band gap of $3.34 \mathrm{eV}$ is obtained between the valence band maximum (VBM) and conduction band minimum (CBM), in good agreement with the results of Sofo et al. [28] and Leenaerts et al. [34]

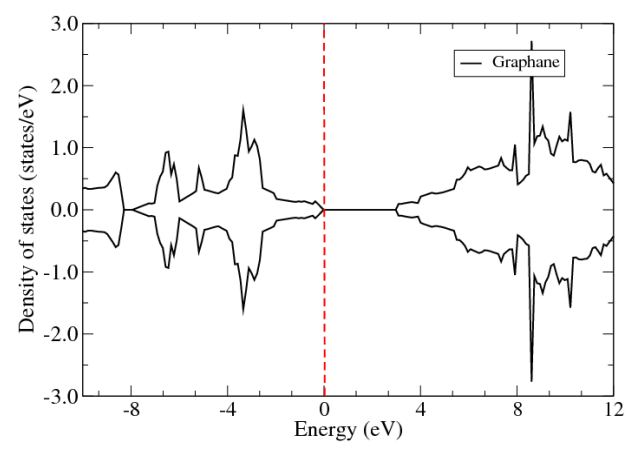

Figure 3: The spin polarized total density for states for graphane. The majority (spin-up) and minority (spin-down) density of states are shown as upper and lower halves of the plot. The Fermi energy is referenced to $0 \mathrm{eV}$ and marked by red dashed vertical line.

Fig. 4 shows various plots of total density of states for hydrogen vacancy configurations $c_{1}$ (row 1), $c_{3}$ (row 2) and $c_{7}$ (row 3 ) that have one, two and three localized unpaired electrons respectively. In each plot, the majority (spin-up) and minority (spin-down) density of states are presented. For each vacancy configuration, we present the density of states plots in charge states $q=-1$ (column 1), 0 (column 2), +1 (column 3). All of the plots show that the ground state of these configurations is spin polarized, with the donor states within the band gap.

Although configurations $c_{1}, c_{3}$ and $c_{7}$ have unpaired electrons and are energetically unfavourable in the neutral charge state $(q=0)$, their spin polarized density of states show an interesting feature at the vicinity of the Fermi level that could make these configurations suitable for spin-based de- 
vices. A sharp peak appears at the Fermi level in the spin-up density of states, with the absence of spin down density of states at the Fermi level. This reveals that the unpaired electrons in the hydrogen vacancies induce a half-metallic character in the system. Therefore the unpaired electrons in these configurations $c_{1}, c_{3}$ and $c_{7}$ produce magnetic moments of $1 \mu_{B}$, $2 \mu_{B}$ and $3 \mu_{B}$ respectively, as shown on Table 2 . It is also interesting to note that each unpaired electron localized on dehydrogenated carbon atom in graphane contributes $1 \mu_{B}$ to the system. An isolated $H$ vacancy represents an unpaired electron in the dangling bond extending from the carbon atom, producing a magnetic moment of $1 \mu_{B} \cdot[38,39]$ This finding is in agreement with Gheeraert et al. [56] who stated that the calculated integral number of Bohr magnetons $(1.0 \mu B)$ is necessary for half-metallic ferromagnetic ordering to occur. Configurations that are able to avoid sublattice imbalance due to pairing of electron are non-magnetic ground state with no magnetic moment (see Table 2), therefore are not charged in this study.

We see in Fig. 4 column 1 that the addition of charge state $q=-1$ into $\mathrm{c}_{1}$ shifts the induced donor states as well as the Fermi level toward the CBM. It is clear that the negatively charged $c_{1}$ is an n-type system with an excess of electrons that are ready to flow into the conduction band. When charging configuration $\mathrm{c}_{1}$ with $q=+1$ as shown in column 2 , the induced donor states become acceptor and shift towards the VBM. The Fermi level also shifts towards the VBM revealing the electronic deficiency, and thus the positively charged $c_{1}$ is a p-type material. Since the shifting of induced states and Fermi level are not of the same magnitude, therefore the effect of charging induces a half-metallic to metallic transition as shown in Fig. 4. This might 
be a disadvantage for spintronics applications. Similar trend is observed in the DOS for $c_{3}$ and $c_{7}$.


Figure 4: The spin polarized total density of states of graphane with hydrogen vacancy configurations $\mathrm{c}_{1}$ (row 1 ), $\mathrm{c}_{2}$ (row 2 ) and $\mathrm{c}_{7}$ (row 3 ) that have one, two and three unpaired electrons respectively. The plots are presented in charge states $q=-1$ (column 1), 0 (column 2), +1 (column 2).

Finally, we investigate the effect of charge injection on the induced magnetic moment in configurations $\mathrm{c}_{1}, \mathrm{c}_{3}$ and $\mathrm{c}_{7}$. In Table 3 , we observe a consistent reduction of a magnetic moment for each configuration. For instance in configuration $c_{1}$, the addition of an electron giving a 0 to -1 transition reduces the magnetic moment from $1.00 \mu_{B}$ to $0.18 \mu_{B}$, and also the transition 
from 0 to +1 switches the magnetic moment from $1.00 \mu_{B}$ to $0.29 \mu_{B}$. As mentioned earlier, the creation of $c_{1}$ generates a dangling bond on dehydrogenated carbon atom due to localized unpaired electron that is responsible for a magnetic moment of $1.00 \mu_{B}$. Therefore, the injection of an electron $(-1)$ saturates the hydrogen vacancy-induced dangling bond during electronic pairing, whereas in the case of the withdrawal of electron $(+1)$, the easy target will be the localized electron that induces the state within the band gap. Looking at the configurations $c_{3}$ and $c_{7}$ that have excess of two and three unpaired electrons respectively, we find that the injection of charge states -1 or +1 reduces the magnetic moment by the magnitude of about $1.00 \mu_{B}$.

\section{Conclusions}

This paper presents the characterization of the possible hydrogen vacancy configurations located on a single hexagonal ring of graphane using the DFT approach. The hydrogen vacancy configurations that have a complete pairing of localized electrons, minimizing sublattice imbalance, have relatively low formation energies, and are wide band gap materials. The other uncharged configurations that always have unpaired electrons have relatively higher formation energies, and induce spin polarized states within the band gap of graphane system. These hydrogen vacancy configurations have halfmetallic magnetic character, with notable magnetic moments that have a great potential for spintronics applications. Charging these configurations, we noted that the -1 charged ( +1 charged) vacancy configurations are more favourable in the p-type (n-type) region respectively. The deep acceptor and donor levels associated with $(0 /-1)$ and $(0 /+1)$ transitions in the band gap 
Table 3: The calculated magnetic moment $m\left(\mu_{B}\right)$ of all hydrogen vacancy configurations considered in a graphane supercell, in charge states $q=-1,0,1$. The MM, HMM and NM represent metallic-magnetic, half-metallic magnetic and non-magnetic ground states respectively.

\begin{tabular}{cccc}
\hline \hline Configurations & \multicolumn{3}{c}{ Charge states } \\
& -1 & 0 & 1 \\
\hline $\mathrm{c}_{1}$ & $0.18(\mathrm{MM})$ & $1.00(\mathrm{HMM})$ & $0.29(\mathrm{MM})$ \\
$\mathrm{c}_{2}$ & & $0.00(\mathrm{NM})$ & \\
$\mathrm{c}_{3}$ & $1.12(\mathrm{MM})$ & $2.00(\mathrm{HMM})$ & $1.01(\mathrm{MM})$ \\
$\mathrm{c}_{4}$ & $0.96(\mathrm{MM})$ & $2.00(\mathrm{HMM})$ & $1.03(\mathrm{MM})$ \\
$\mathrm{c}_{5}$ & $0.29(\mathrm{MM})$ & $1.00(\mathrm{HMM})$ & $0.01(\mathrm{NM})$ \\
$\mathrm{c}_{6}$ & $0.21(\mathrm{MM})$ & $1.00(\mathrm{HMM})$ & $0.09(\mathrm{MM})$ \\
$\mathrm{c}_{7}$ & $2.00(\mathrm{MM})$ & $3.00(\mathrm{HMM})$ & $2.02(\mathrm{MM})$ \\
$\mathrm{c}_{8}$ & & $0.00(\mathrm{NM})$ & \\
$\mathrm{c}_{9}$ & $1.21(\mathrm{MM})$ & $2.00(\mathrm{HMM})$ & $1.01(\mathrm{MM})$ \\
$\mathrm{c}_{10}$ & & $0.00(\mathrm{NM})$ & \\
$\mathrm{c}_{11}$ & $0.15(\mathrm{MM})$ & $1.00(\mathrm{NM})$ & $0.00(\mathrm{NM})$ \\
$\mathrm{c}_{12}$ & & $0.00(\mathrm{NM})$ & \\
\hline \hline
\end{tabular}

are noted. This therefore suggests that these vacancy defects may be suitable for high temperature devices exploitation. For low temperature application, higher vacancy concentrations would be required in order to introduce more in-gap donor states which would be easily accessible by electron tunneling between the states. Due to charge doping, the desired half-metallic character is lost. The addition of charge $q=-1(q=+1)$ shifts the induced donor states as well as the Fermi level toward the CBM (VBM) while the induced 
magnetic moment is reduced.

\section{Acknowledgments}

The authors would like to thank the University of Pretoria and National Research Foundation (NRF) for financial support and computational resources. NC is grateful to the National Institute for Theoretical Physics (NITheP) for support. MPM is grateful to the University of South Africa for financial support. The authors are also grateful to Professor J. Pretorius and Professor W. Meyer for technical assistance.

\section{References}

[1] K. S. Novoselov, A. K. Geim, S. V. Morozov, D. Jiang, Y. Zhang, S. V. Dubonos, I. V. Grigorieva, and A. A. Firsov, Science 306, 666 (2004).

[2] A. K. Geim and K. S. Novoselov, Nature Mater. 6, 183 (2007).

[3] P. R. Wallace, Phys. Rev. 71, 622 (1947).

[4] K. S. Novoselov, A. K. Geim, S. V. Morozov, D. Jiang, Y. Zhang, M. I. Katsnelson, S. V. Dubonos, I. V. Grigorieva, and A. A. Firsov, Nature (London) 438, 197 (2005).

[5] K. S. Novoselov, Z. Jiang, Y. Zhang, S. V. Morozov, H. L. Stormer, U. Zeitler, J. C. Maan, G. S. Boebinger, P. Kim, and A. K. Geim, Science 315, 1379 (2007).

[6] L. Sun, Q. X. Li, H. Ren, H. B. Su, Q. W. Shi, and J. L. Yang, J. Chem. Phys 129, 074074 (2008).

[7] Y. W. Son, M. L. Cohen, and S. G. Louie, Phys. Rev. Lett 97, 216803 (2006). 
[8] M. Y. Han, B. Ozyilmaz, Y. Zhang, and P. Kim, Phys. Rev. Lett 98, 206805 (2007).

[9] F. Ding, Phys. Rev. B 72, 245409 (2005).

[10] R. M. Ribeiro, N. M. R. Peres, J. Coutinho, and P. R. Briddon, Phys. Rev. B 78, 075442 (2008).

[11] G. D. Lee, C. Z. Wang, E. Yoon, N. M. Hwang, D. Y. Kim, and K. M. Ho, Phys. Rev. Lett. 95, 205501 (2005).

[12] E. J. Duplock, M. Scheffler, and P. J. D. Lindan, Phys. Rev. Lett. 92, 225502 (2004).

[13] M. T. Lusk and L. D. Carr, Phys. Rev. Lett. 100, 175503 (2008).

[14] S. C. Pradhan and J. K. Phadikar, Phys. Lett. A 373, 1062 (2009).

[15] P. Lu, Z. Zhang, and W. Guo, Phys. Lett. 373, 3354 (2009).

[16] Y. H. Lu, W. Chen, Y. P. Feng, and P. M. He, J. Phys. Chem. B 113, 2 (2009).

[17] I. Zanella, S. Guerini, S. B. Fagan, J. MendesFilho, and A. G. SouzaFilho, Phys. Rev. B 77, 073404 (2008).

[18] D. W. Boukhvalov, M. I. Katsnelson, and A. I. Lichtenstein, Phys. Rev. B 77, 035427 (2008).

[19] S. Lebegue, M. Klintenberg, O. Eriksson, and M. I. Katsnelson, Phys. Rev. B 79, 245117 (2009).

[20] D. W. Boukhvalov and M. I. Katsnelson, Phys. Rev. B 78, 085413 (2008). 
[21] N. Z. Lu, Z. Y. Li, and J. L. Yang, J. Phys. Chem. C 113, 16741 (2009).

[22] J. A. Yan, L. Xian, and M. Y. Chou, Phys. Rev. Lett. 103, 086802 (2009).

[23] X. S. Wu, M. Sprinkle, X. B. Li, F. Ming, C. Berger, and W. A. de Heer, Phys. Rev. Lett. 101, 026801 (2008).

[24] I. Jung, D. A. Dikin, R. D. Piner, and R. S. Ruoff, Nano Lett. 8, 4283 (2008).

[25] Z. Luo, P. M. Vora, E. J. Mele, A. T. C. Johnson, and J. M. Kikkawa, Appl. Phys. Lett. 94, 111909 (2009).

[26] R. Balog, B. Jorgensen, L. Nilsson, M. Andersen, E. Rienks, M. Bianchi, M. Fanetti, E. Laegsgaard, A. Baraldi, S. Lizzit, Z. Sljivancanin, F. Besenbacher, B. Hammer, T. G. Pedersen, P. Hofmann, and L. Hornekaer, Nature Mater. 9, $315(2010)$.

[27] A. Savchenko, Science 323, 589 (2009).

[28] J. O. Sofo, A. S. Chaudhari, and G. D. Barber, Phys. Rev. B 75, 153401 (2007).

[29] D. C. Elias, R. R. Nair, T. M. G. Mohiuddin, S. V. Morozov, P. Blake, M. P. Halsall. A. C. Ferrari, D. W Boukhvalov, M. I. Katsnelson, A. K. Geim, and K. S. Novoselov, Science. 323, 610 (2009).

[30] F. Withers, M. Dubois, and A. K. Savchenko, Phys. Rev. B 82, 073403 (2010).

[31] S. H. Cheng, K. Zou, F. Okino, H. R. Gutierrez, A. Gupta, N. Shen, P. C. Eklund, J. O. Sofo, and J. Zhu, Phys. Rev. B 81, 205435 (2010).

[32] R. Paupitz, P. A. S. Autreto, S. B. Legoas, S. Goverapet Srinivasan, A. C. T. van Duin, and D. S. Galvao, Nanotechnology 24, 0357062013. 
[33] M. H. F. Sluiter and Y. Kawazoe, Phys. Rev. B 68, 085410 (2003).

[34] O. Leenaerts, H. Peelaers, A. D. Hernandez-Nieves, B. Partoens, and F. M. Peeters, Phys. Rev. B 82, 195436 (2010).

[35] H. Sahin, O. Leenaerts, S. K. Singh, and F.M. Peeters, WIREs: Comp. Mol. Sci. 5, 255 (2015).

[36] P. Chandrachud, B. S. Pujari, S. Haldar, B. Sanyal, and D. G. Kanhere, J. Phys. Condens. Matter 22, 465502 (2010).

[37] H. Sahin, C. Ataca, and S. Ciraci, Appl. Phys. Lett. 95, 222510 (2009).

[38] Chi-Kai Yang, Carbon, 48, 3901 (2010).

[39] B. Wu and C. Yang, APL. Adv. 2, 012173 (2012).

[40] B.S. Pujari and D.G. Kanhere, J. Phys. Chem. C 113, 21063-7 (2009).

[41] J. Berashevich and T. Chakraborty, Nanotechnology 21, 355201 (2010).

[42] G. Kresse and J. Hafner, Phys. Rev. B 47, 558 (1993).

[43] J. P. Perdew, K. Burke, and M. Ernzerhof, Phys. Rev. Lett. 77, 3865 (1996).

[44] P. E. Blochl, Phys. Rev. B 50, 17953 (1994).

[45] H. J. Monkhorst and J. D. Pack, Phys. Rev. B 13, 5188 (1976).

[46] M. Methfessel and A. T. Paxton, Phys. Rev. B 40, 3616 (1989).

[47] A. A. Dzhurakhalov and F. M. Peeters, Carbon 49, 3258 (2011).

[48] S. Casolo, O. M. Lovvik, R. Martinazzo, and G. F. Tantardini, J. Chem. Phys. 130, 054704 ( 2009). 
[49] G. Makov and M. C. Payne, Phys. Rev. B 51, 4014 (1995).

[50] C. G. Van de Walle and J. Neugebauer, J. Appl. Phys. 95, 3851 (2004).

[51] S. Lany and A. Zunger, Phys. Rev. B 78, 235104 (2008).

[52] E. Ertekin, L. K. Wagner, and J.C. Grossman, Phys. Rev. B 87, 155210 (2013).

[53] R. O. Jones and O. Gunnarson, Rev. Mod. Phys 61, 689 (1989).

[54] A. Savchenko, Science 323, 589 (2009).

[55] R.E. Mapasha, A.M. Ukpong, and N. Chetty, Phys. Rev. B 85, 205402 (2012).

[56] E. Gheeraert, S. Koizumi, T. Teraji, H. Kanda, and M. Nesladek, Diamond Relat. Mater. 9, 948 (2000). 